Am J Nephrol 2021;52:949-957

DOI: $10.1159 / 000519758$
Received: May 13, 2021

Accepted: September 6, 2021

Published online: December 7, 2021

\title{
Disparities in Chronic Kidney Disease Progression by Medicare Advantage Enrollees
}

\author{
Clarissa Jonas Diamantidis ${ }^{a, b}, c$ Lindsay Zepel ${ }^{c, d}$ Virginia Wang ${ }^{a, c, e}$ \\ Valerie A. Smith ${ }^{c,}$ e Sarah Hudson Scholle ${ }^{g}$ Loida Tamayo $^{f}$ \\ Matthew L. Maciejewskia, ${ }^{a}, \mathrm{e}$
}

${ }^{a}$ Division of General Internal Medicine, Department of Medicine, Duke University School of Medicine, Durham, NC, USA; ${ }^{b}$ Division of Nephrology, Department of Medicine, Duke University School of Medicine, Durham, NC, USA; 'Department of Population Health Sciences, Duke University School of Medicine, Durham, NC, USA; ${ }^{d}$ OptumLabs Visiting Fellow, Cambridge, MA, USA; ${ }^{\circ}$ Center for Health Services Research in Primary Care, Durham Veterans Affairs Medical Center, Durham, NC, USA; ${ }^{\mathrm{f} C e n t e r s ~ f o r ~ M e d i c a r e ~ \& ~ M e d i c a i d ~ S e r v i c e s, ~ B a l t i m o r e, ~ M D, ~ U S A ; ~}{ }^{9}$ National Committee for Quality Assurance, Washington, DC, USA

\section{Keywords}

Medicare · Beneficiary · Chronic kidney disease · Disparities · Progression

\begin{abstract}
Introduction: The prevalence of chronic kidney disease (CKD) in Medicare beneficiaries has quadrupled in the past 2 decades, but little is known about risk factors affecting the progression of CKD. This study aims to understand the progression in Medicare Advantage enrollees and whether it differs by provider recognition of CKD, race and ethnicity, or geographic location. In a large cohort of Medicare Advantage (MA) enrollees, we examined whether CKD progression, up to 5 years after study entry, differed by demographic and clinical factors and identified additional risk factors of CKD progression. Methods: In a cohort of 1,002,388 MA enrollees with CKD stages 1-4 based on 2013-2018 labs, progression was estimated using a mixed-effects model that adjusted for demographics, geographic location, comorbidity, urine albumin-to-creatinine ratio, clinical recognition via diagnosed
\end{abstract}

CKD, and time-fixed effects. Race and ethnicity, geographic location, and clinical recognition of CKD were interacted with time in 3 separate regression models. Results: Mean (median) follow-up was 3.1 (3.0) years. Black and Hispanic MA enrollees had greater kidney function at study entry than other beneficiaries, but their kidney function declined faster. MA enrollees with clinically recognized CKD had estimated glomerular filtration rate levels that were 18.6 units (95\% confidence interval [CI]: 18.5-18.7) lower than levels of unrecognized patients, but kidney function declined more slowly in enrollees with clinical recognition. There were no differences in CKD progression by geography. After removal of the race coefficient from the eGFR equation in a sensitivity analysis, kidney function was much lower in all years among Black MA enrollees, but patterns of progression remained the same. Discussion/Conclusions: These results suggest that patients with clinically recognized CKD and racial and ethnic minorities merit closer surveillance and management to reduce their risk of faster progression.

(c) 2021 S. Karger AG, Basel karger@karger.com

www.karger.com/ajn
(C) 2021 S. Karger AG, Basel
Correspondence to:

Matthew L. Maciejewski, matthew.maciejewski@duke.edu 


\section{Introduction}

Chronic kidney disease (CKD) is a costly and increasingly prevalent condition that increases patient risk for cardiovascular disease, end-stage renal disease (ESRD), and death [1]. Documented CKD prevalence in Medicare beneficiaries has steadily increased from $2.7 \%$ in 2000 to $10.0 \%$ in 2011 and $11.0 \%$ in 2014, with stage 3 CKD being the fastest growing subgroup [2]. Studies to identify distinct trajectories for CKD progression and risk factors of decline in kidney function have proliferated in recent years [3-5]. A prior study identified 4 trajectories of CKD progression in the 2 years prior to dialysis initiation, where a higher estimated glomerular filtration rate (eGFR) indicates better kidney function: (1) minimal decline in those with advanced CKD (eGFR $<30 \mathrm{~mL} /$ $\mathrm{min} / 1.73 \mathrm{~m}^{2}, 67 \%$ of cohort); (2) linear decline in those with moderate CKD (eGFR 30-59 mL/min/1.73 m², 25\% of cohort); (3) rapid linear decline in those with early CKD (eGFR $>60 \mathrm{~mL} / \mathrm{min} / 1.73 \mathrm{~m}^{2}, 9.5 \%$ of cohort); and (4) nonlinear catastrophic decline (3.1\% of cohort) [6].

Complicating models of CKD progression is prior work indicating that $\mathrm{CKD}$ progression patterns vary by demographic and social risk factors, such that men progress faster than women [7-9], Black patients progress faster than White patients [10], younger patients progress faster than older patients $[9,10]$, and patients with diabetes progress faster than those without diabetes [10]. However, most of these studies have been on small $(n<10,000)$ US-based cohorts, in Veteran cohorts that are predominantly male, or in non-Medicare populations due to the lack of availability of lab values associated with Medicare beneficiaries [11]. Furthermore, most studies were based on cohorts with CKD stages 3-4 [12], so patient factors that may explain variation in CKD progression for patients in earlier CKD stages is less understood.

There is a need to identify additional risk factors of CKD progression, such as lack of diagnoses codes indicating CKD that is significantly underrecognized in Medicare fee-for-service (FFS) patients [13] and has not been examined in prior studies of CKD progression. The purpose of this study was to characterize CKD progression in a large cohort of Medicare Advantage (MA) enrollees from 2013 to 2018 and examine whether CKD progression differed by critical demographic and clinical factors to determine whether the associations for established risk factors generalize to MA patients. Risk factors of race, ethnicity, and geography were chosen because disparities in these immutable patient factors have been found in numerous evaluations of care quality and patient out- comes [14]. Furthermore, prior work suggests that Black and Hispanic individuals with CKD have faster progression than their White counterparts $[15,16]$. We further examined CKD recognition as a modifying variable because CKD is significantly underdiagnosed [13], yet the association between clinical recognition and CKD progression has not been previously examined. It is important to examine CKD progression in MA enrollees specifically because service-level selection bias remains [17, 18] despite selection decreasing in MA overall $[19,20]$. Identification of novel factors of CKD progression may enable the refinement to the existing prediction models [8] that are gradually being used by providers in routine clinical practice [21].

\section{Methods}

\section{Data Source and Cohort}

This study used the OptumLabs ${ }^{\circledR}$ Data Warehouse (OLDW) database. The OLDW contains longitudinal health information on commercial and MA enrollees, representing a diverse mixture of ages, race and ethnicities, and geographical regions across the USA [22]. Specifically, deidentified administrative claims data (medical and pharmacy claims) linked with laboratory results, medical benefit design information, and death information were obtained from the OLDW database to form the cohort of individuals with CKD.

The initial population of MA enrollees in 2013-2018 included $6,167,895$ individuals with medical coverage. We then excluded enrollees $(n=5,165,567)$ who died prior to $2013(n=8,972)$, lacked serum creatinine or urinary albumin-to-creatinine (UACR) lab values ( $n=3,492,602)$, or did not have 2 serum creatinine values at least 90 days apart indicating CKD stages 1-5 (1,654,437; Fig. 1). We further restricted the remaining sample, removing those unable to have CKD progression tracked because they had an eGFR value $<15 \mathrm{~mL} / \mathrm{min} / 1.73 \mathrm{~m} \mathrm{[2]}$, had diagnosed ESRD or were on dialysis, or had a kidney transplant $+/-30$ days from baseline $(n=$ 9,556). The final analytic sample included 1,002,328 MA enrollees with CKD whose CKD progression was followed from the date of the second qualifying creatinine lab date (which was used for baseline eGFR determination) until they were censored at the earliest of ESRD diagnosis, kidney transplant, MA disenrollment, dialysis initiation, or death or the last creatinine lab date. The vast majority $(92 \%)$ of patients in the final analytic sample had follow-up end on December 31,2018, or were censored due to disenrollment from MA (online suppl. Table 1; for all online suppl. material, see www. karger.com/doi/10.1159/000519758).

\section{Covariates}

Covariates considered in the analysis of CKD progression included demographic characteristics (age, sex, race, and ethnicity), geographic location (metropolitan, micropolitan, and small/rural), UACR, chronic conditions as measured by the Charlson comorbidity index, and a measure indicating whether a patient had their CKD clinically recognized via the presence of diagnoses codes for CKD. Geographic location was defined based on rural-urban com-
Diamantidis/Zepel/Wang/Smith/ Hudson Scholle/Tamayo/Maciejewski 


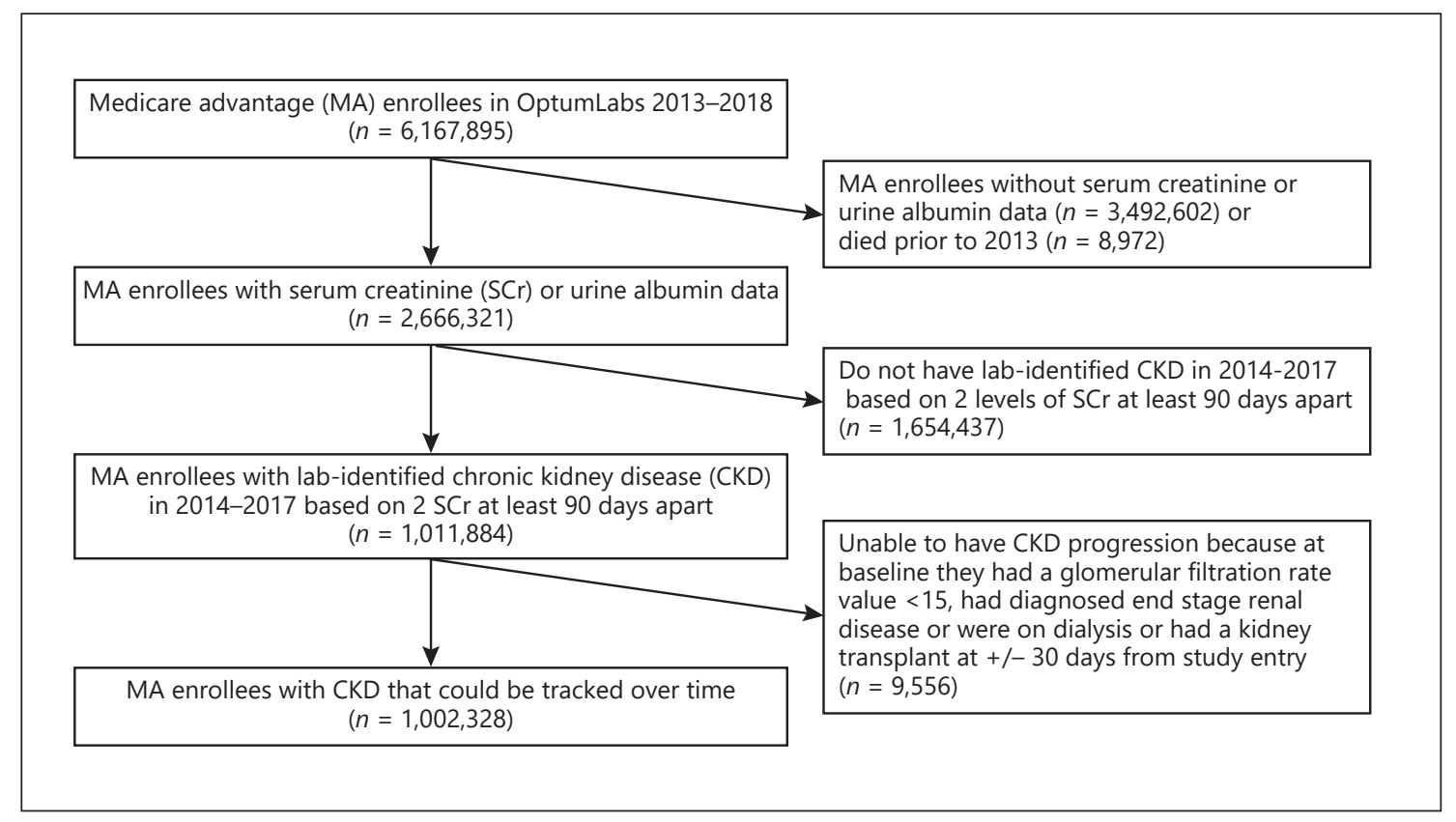

Fig. 1. STROBE figure of the analytic cohort. *Beneficiaries with qualifying diagnosis of CKD but no qualifying labs indicating CKD includes patients with only 1 qualifying lab; $2+$ qualifying labs that are $<90$ days apart; labs processed by a different lab vendor than the one providing these data, by in-house labs, or through point-of-care testing; or no labs in 2013-2018 processed by any lab vendor, in-house lab, or point-of-care testing. CKD, chronic kidney disease.

muting area (RUCA) codes [23]. The categories small and rural were combined due to small sample size. OptumLabs provides enrollees' first, middle, and last names and zip code information to a nationally recognized supplier of consumer marketing data that cross-references these data elements to public data and derives predictive data to generate a weighted prediction of race and ethnicity from over 180 ethnicities. This proprietary method is consistent with other approaches to impute race and ethnicity based on geocoding, name analysis, or a combination of the two. OptumLabs provides researchers a deidentification compliant variable that identifies the most likely prediction, which is aggregated to label individuals as White, Black, Hispanic, Asian, or others/unknown. Clinically recognized CKD was defined as a diagnosis (ICD-9 of 585.x or ICD-10 of N18.x) between 12 months before or 12 months after baseline. Covariates were of 2 types: (1) risk factors of specific interest to understand whether CKD progression differed by subgroups of race and ethnicity, geographic location, or clinical recognition of CKD and (2) control variables of secondary interest that were entered as main effects in the regression analysis.

\section{Outcomes}

The outcome of interest was the rate of CKD progression as captured by eGFR decline over the 5-year study period. eGFR was calculated from the CKD-EPI equation [24] using serum creatinine values measured anytime between baseline in 2014-2017 until the earlier of the patient's last creatinine lab date or censoring date. As the CKD-EPI equation dichotomizes race into Black or non-Black, patients with Hispanic, Asian, or missing race and ethnicity data were assigned to non-Black to calculate eGFR.

\section{Statistical Analysis}

Study cohort characteristics were summarized using mean and standard deviation for continuous variables and frequency and percent for categorical variables. To examine patient factors associated with CKD progression, we estimated a mixed-effects model with a random intercept and random slope, specifying time since baseline as a linear and quadratic effect since we hypothesized that progression might not be linear over time. This approach allowed us to estimate overall mean trajectories and individual trajectories with enough flexibility to reflect CKD progression during an individual's entire follow-up. This method generates estimates of kidney function over time that are less biased by nonrandom attrition than alternative methods [25]. We adjusted for time measured as years since baseline, demographic characteristics, geographic location, Charlson comorbidity index, UACR at baseline, year of baseline (e.g., study entry), and clinical recognition of CKD via diagnoses code. A restricted model excluding interactions was generated to report main effects of CKD progression.

To assess whether CKD progression differed by race and ethnicity, geographic location, or clinical recognition of CKD via diagnosis code, these 3 risk factors were interacted with time in a set of "full" specification in 3 separate regression models (one for race and ethnicity interactions, one for geography interactions, and one for clinical recognition interactions). Given the current debate in use of a race coefficient in the calculation of eGFR [26], we then performed a post hoc sensitivity analysis using calculated eGFR without a race coefficient. This analysis was done on a cohort of 989,703 enrollees because the recalculation of eGFR disqualified 12,625 enrollees from the sample (change in baseline eGFR date [ $n$ 
Table 1. Descriptive statistics of medicare advantage enrollees at baseline (2014-2017)

\begin{tabular}{|c|c|}
\hline & $\begin{array}{l}\text { Overall } \\
(n=1,002,328)\end{array}$ \\
\hline \multicolumn{2}{|l|}{ Stage of CKD identified in lab data, $n(\%)$} \\
\hline Stage 1 & $4,602(0.5)$ \\
\hline Stage 2 & $650,275(64.9)$ \\
\hline Stage 3 & $317,605(31.7)$ \\
\hline Stage 4 & $29,846(3.0)$ \\
\hline Age, mean (SD), years & $73.4(7.9)$ \\
\hline \multicolumn{2}{|l|}{ Age group, $n(\%)$} \\
\hline $18-44$ years & $3,060(0.3)$ \\
\hline $45-54$ years & $15,801(1.6)$ \\
\hline $55-64$ years & $63,323(6.3)$ \\
\hline $65-74$ years & $486,893(48.6)$ \\
\hline$\geq 75$ years & $433,251(43.2)$ \\
\hline Female gender, $n(\%)$ & $580,933(58.0)$ \\
\hline \multicolumn{2}{|l|}{ Race and ethnicity } \\
\hline White & $661,122(66.0)$ \\
\hline Black & $122,882(12.3)$ \\
\hline Hispanic & $120,877(12.1)$ \\
\hline Asian & $42,379(4.2)$ \\
\hline Unknown & $55,068(5.5)$ \\
\hline \multicolumn{2}{|l|}{ Geographic location, $n(\%)$} \\
\hline Metropolitan & $889,018(88.7)$ \\
\hline Micropolitan & $63,558(6.3)$ \\
\hline Rural/small & $49,442(4.9)$ \\
\hline Unknown & $310(0.0)$ \\
\hline Diabetes, $n(\%)$ & $403,680(40.3)$ \\
\hline Hypertension, $n(\%)$ & $807,978(80.6)$ \\
\hline Charlson Comorbidity Index, mean (SD) & $2.2(2.3)$ \\
\hline Outpatient nephrologist visit at baseline, $n(\%)$ & $89,057(8.9)$ \\
\hline Outpatient primary care physician visit at baseline, $n(\%)$ & $970,334(96.8)$ \\
\hline \multicolumn{2}{|l|}{ Clinical recognition of CKD via diagnoses \pm 12 months from baseline, } \\
\hline \multicolumn{2}{|l|}{ Urinary albumin-to-creatinine values } \\
\hline$<30$ & $38,635(3.9)$ \\
\hline $30-300$ & $16,432(1.6)$ \\
\hline$>300$ & $3,805(0.4)$ \\
\hline Missing & $943,456(94.1)$ \\
\hline \multicolumn{2}{|l|}{ Year of study entry } \\
\hline 2014 & $299,538(29.9)$ \\
\hline 2015 & $185,358(18.5)$ \\
\hline 2016 & $220,991(22.0)$ \\
\hline 2017 & $296,441(29.6)$ \\
\hline
\end{tabular}

$=12,190]$ and re-categorization to stage 5 CKD $[n=437])$. The study was approved as exempt by the Institutional Review Board of Duke University.

\section{Results}

\section{Characteristics of Analytic Cohort}

The cohort of 1,002,328 MA enrollees had a mean (median) follow-up of 3.1 (3.0) years. Enrollees excluded from analysis $(n=5,165,567)$ were younger, with a larger proportion of "unknown" race, and rural geographic location than those included in the 1,002,328 patients in the final cohort (online suppl. Table 2). Nearly two-thirds of the final cohort $(64.9 \%)$ had stage $2 \mathrm{CKD}$ at baseline (eGFR $60-<90 \mathrm{~mL} / \mathrm{min} / 1.73 \mathrm{~m}^{2}$ ), $31.7 \%$ had stage 3 at baseline (eGFR $30-<60 \mathrm{~mL} / \mathrm{min} / 1.73 \mathrm{~m}^{2}$ ), and the remainder had stage 4 (3.0\%) (eGFR $15-<30 \mathrm{~mL} / \mathrm{min} / 1.73$ $\left.\mathrm{m}^{2}\right)$ or stage $1(0.5 \%)$ CKD $\left(\mathrm{eGFR}>90 \mathrm{~mL} / \mathrm{min} / 1.73 \mathrm{~m}^{2}\right.$ with UACR $\geq 30 \mathrm{mg} / \mathrm{g}$ ). The average age was $73.4,58 \%$ were female, $66 \%$ were White, $12.3 \%$ were Black, $12.1 \%$ were Hispanic, and $4.2 \%$ were Asian (Table 1 ). A vast ma- 
Fig. 2. Annual decline in kidney function in milliliters per minute per 1.73 square meters by race/ethnicity. Numbers above represent slopes (annual decline) of kidney function for each group. Note that $p$ value $<0.0001$ for interaction of race/ethnicity and year and year-squared.
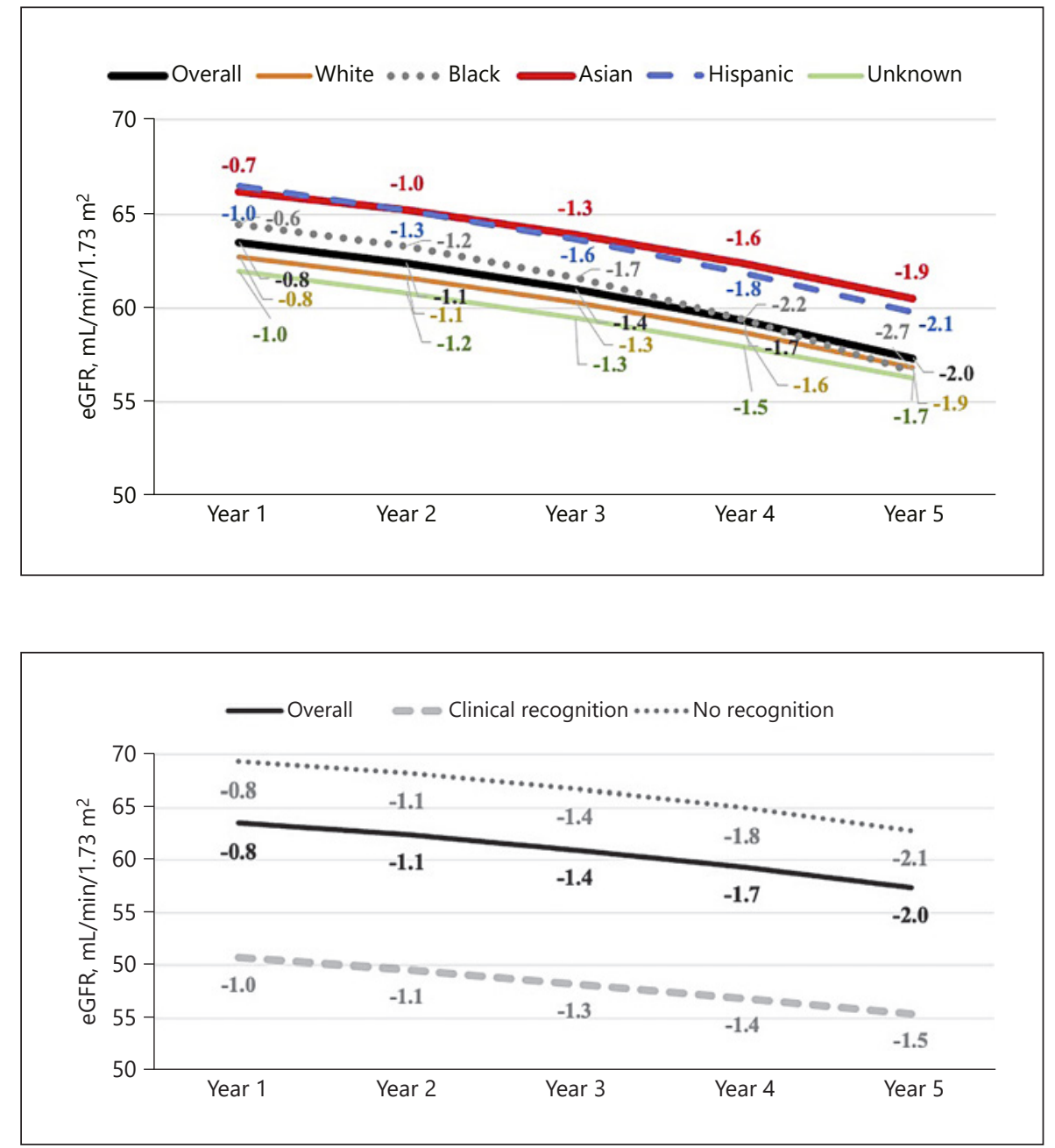

Fig. 3. Annual decline in kidney function in milliliters per minute per 1.73 square meters by clinical recognition. Numbers above represent slopes (annual decline) of kidney function for each group. Note that $p$ value $<0.0001$ for interaction of clinical recognition and year and year-squared. jority (88.7\%) lived in metropolitan areas, and the mean Charlson score was 2.2, with $40.3 \%$ having diagnosed diabetes and $80.6 \%$ having diagnosed hypertension. Approximately, $9 \%$ saw a nephrologist within a year before or year after baseline, $96.8 \%$ saw a PCP, and $26 \%$ had their CKD clinically recognized via diagnoses claims. Nearly all (94.1\%) had missing UACR values at baseline.

\section{Factors Associated with CKD Progression}

The median number of available serum creatinine measurements was 4 across race/ethnicity, with the exception of "unknown race" which had a median of 3 (online suppl. Table 3). All patient factors included in the regression model were significant, with CKD progression exhibiting a slightly nonlinear trend as indicated by the significant coefficient for the quadratic time term (online suppl. Table 4). MA enrollees aged $<65$ years had higher eGFR than MA enrollees aged 65-74 years, while those aged 75 years or higher had lower eGFR $(-5.58 \mathrm{~mL} /$ $\mathrm{min} / 1.73 \mathrm{~m}^{2}, 95 \%$ confidence interval [CI]: $\left.-5.64,-5.53\right)$; women had lower eGFR than men $(-1.93 \mathrm{~mL} / \mathrm{min} / 1.73$ $\mathrm{m}^{2}, 95 \%$ CI: $\left.-1.99,-1.88\right)$. Black and Hispanic enrollees had higher eGFR than White enrollees; patients with higher Charlson comorbidity scores (i.e., greater burden of illness) had lower eGFR than patients with lower Charlson scores $\left(-0.43 \mathrm{~mL} / \mathrm{min} / 1.73 \mathrm{~m}^{2} ; 95 \% \mathrm{CI}:-0.44,-0.41\right)$.

Trends in CKD Progression by Race and Ethnicity,

Clinical Recognition, and Geography

Decline in eGFR was observed in all race and ethnicity groups (Fig. 2). Black and Hispanic enrollees had higher eGFR levels at baseline, but faster decline over time than all other racial and ethnic groups 3-5 years after baseline ( $p$ value $<0.0001$ for interactions of year and year [2] by race and ethnicity). In the first year after baseline, Hispanic enrollees had an annual eGFR decline of $-1.0 \mathrm{~mL} /$ 

milliliters per minute per 1.73 square mesent slopes (annual decline) of kidney function for each group. Note that $p$ value $<0.0001$ for interaction of geography and year and year-squared.
Fig. 4. Annual decline in kidney function in ters by urbanicity. Numbers above repre-

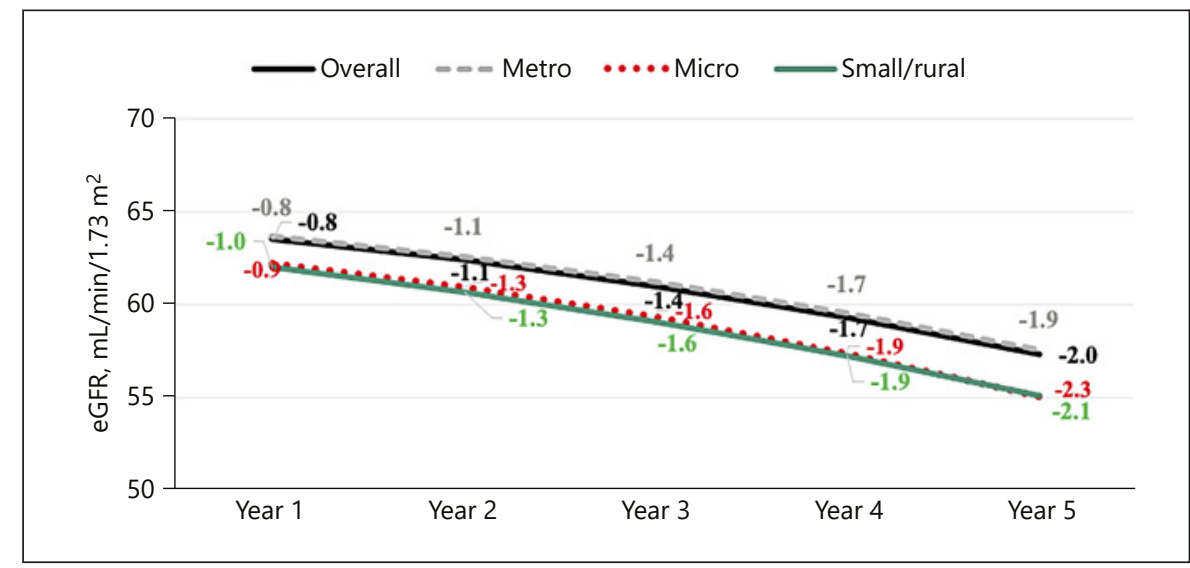

Fig. 5. Annual decline in kidney function in milliliters per minute per 1.73 square meters by race/ethnicity using eGFR without the race coefficient. Numbers above represent slopes (annual decline) of kidney function for each group. Note that $p$ value $<0.0001$ for interaction of race/ethnicity and year and year-squared.

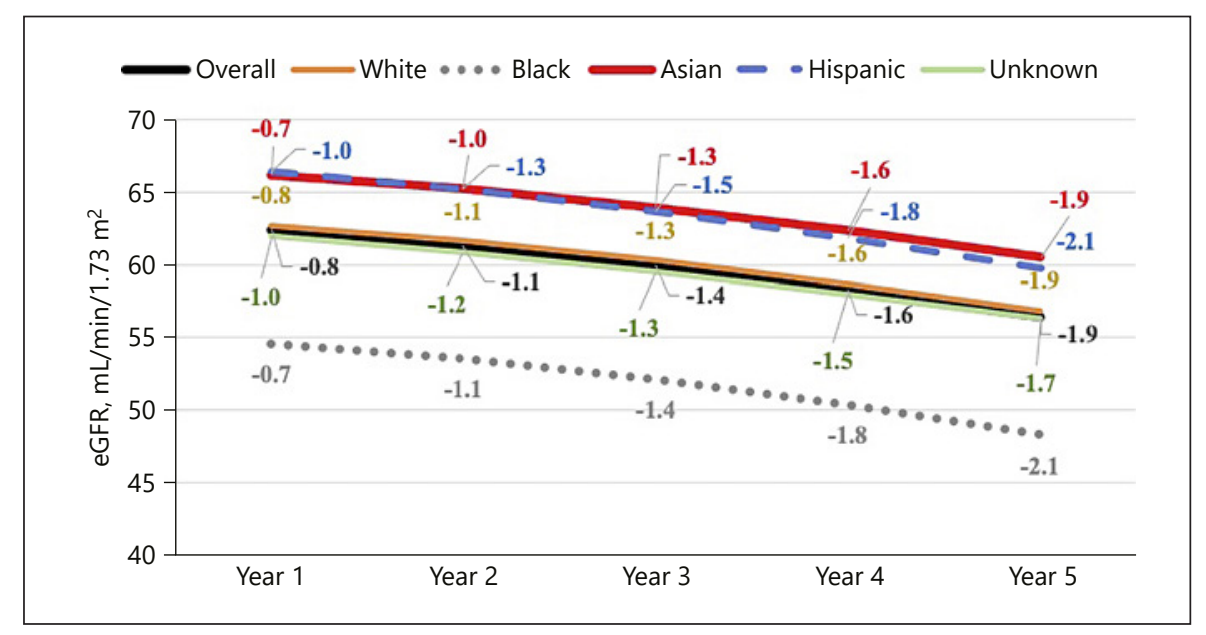

$\min / 1.73 \mathrm{~m}^{2}$ from baseline, Black enrollees had an annual eGFR decline of -0.6 , and White enrollees had an annual eGFR decline of -0.8 . At 3 years after baseline, annual decline was $-1.6 \mathrm{~mL} / \mathrm{min} / 1.73 \mathrm{~m}^{2}$ for Hispanic enrollees, $-1.7 \mathrm{~mL} / \mathrm{min} / 1.73 \mathrm{~m}^{2}$ for Black enrollees, and -1.3 for White enrollees. These differences were magnified 5 years after baseline when annual decline was -2.1 $\mathrm{mL} / \mathrm{min} / 1.73 \mathrm{~m}^{2}$ for Hispanic enrollees, $-2.7 \mathrm{~mL} /$ $\min / 1.73 \mathrm{~m}^{2}$ for Black enrollees, and -1.9 for White enrollees.

MA enrollees with clinical recognition based on administrative coding had slower CKD progression than patients without clinical recognition ( $p$ value $<0.0001$ for interactions). Patients with clinically recognized CKD had lower eGFR levels than patients without code-based clinical recognition (Fig. 3). The eGFR of patients with clinically recognized CKD was $18.6 \mathrm{~mL} / \mathrm{min} / 1.73 \mathrm{~m}^{2}$ lower (95\% CI: 18.5-18.7) than the eGFR of patients without clinical recognition in the first year after baseline and remained significantly lower 5 years after baseline (17.5 units, 95\% CI: 17.3-17.7). While in the first year after baseline, the annual decline was $-1.0 \mathrm{~mL} / \mathrm{min} / 1.73 \mathrm{~m}^{2}$ for clinically recognized enrollees and $-0.8 \mathrm{~mL} / \mathrm{min} / 1.73 \mathrm{~m}^{2}$ for unrecognized enrollees, and this increased to an annual decline of $-1.5 \mathrm{~mL} / \mathrm{min} / 1.73 \mathrm{~m}^{2}$ for clinically recognized enrollees and $-2.1 \mathrm{~mL} / \mathrm{min} / 1.73 \mathrm{~m}^{2}$ for unrecognized enrollees 5 years after baseline.

Baseline kidney function was similar for patients in metropolitan, micropolitan, and small/rural areas and had similar annual decline in kidney function in the first year of study entry (Fig. 4). Five years after study entry, MA enrollees in metropolitan areas had slower annual decline $\left(-1.9 \mathrm{~mL} / \mathrm{min} / 1.73 \mathrm{~m}^{2}\right)$ than enrollees in micropolitan areas $\left(-2.3 \mathrm{~mL} / \mathrm{min} / 1.73 \mathrm{~m}^{2}\right)$ or small $/ \mathrm{ru}$ ral areas $\left(-2.1 \mathrm{~mL} / \mathrm{min} / 1.73 \mathrm{~m}^{2}, p\right.$ value $<0.0001$ for interactions).

\section{Sensitivity Analysis}

After removal of the race coefficient from the eGFR equation $(n=989,703)$, eGFR levels shifted down in all 
years for Black enrollees (Fig. 5; online suppl. Table 5). That is, mean eGFR for Black patients was 1.63 points higher at baseline than White patients when including the race coefficient in the eGFR calculation (Fig. 2) but becomes 8 points lower than White patients at baseline when removing the race coefficient from the eGFR calculation. Overall, annual decline of eGFR did not differ from the primary analysis after removal of the race coefficient for all groups. There were no meaningful differences in annual eGFR decline by clinical recognition or urbanicity and clinical recognition with exclusion of the race coefficient (data not shown).

\section{Discussion}

This is the first study to examine CKD progression in a longitudinal cohort of Medicare Advantage enrollees with stages 1-4 (96.6\% had stages 2 or 3$)$ who were followed for an average of 3 years. We found that Black and Hispanic MA enrollees had faster kidney function decline over time even after controlling for comorbidity than other beneficiaries, despite having greater kidney function at baseline in the primary analysis. One additional reason for this finding may be that these initial eGFR estimates are reflective of hyperfiltration, which is associated with an accelerated decline over time. Although we found a reduction in baseline eGFR with exclusion of the race coefficient, we did not observe a significant difference in kidney function decline. Overall, this result is consistent with prior work indicating an accelerated rate of eGFR decline in ethnic and racial minorities compared to Whites [15], but is the first do so in an MA population or in a sample of over 1 million patients obtained by linking laboratory and pharmacy data [11].

Disparities in CKD progression among MA enrollees are concerning because ethnic and racial minorities are disproportionately affected by CKD and suffer worse clinical outcomes than nonminorities, including progression to ESRD, incident cardiovascular disease, and death $[16,27]$. The current analysis validates recent initiatives in the nephrology community to target risk stratification and treatment strategies on predicted risk, rather than on current eGFR alone $[8,28]$. Early mitigation strategies such as medication management with renin-angiotensin aldosterone system blockade for proteinuria reduction and referral to specialty care based on predicted progression can attenuate the increased risk of adverse outcomes experienced by ethnic and racial minorities with CKD [29]. Furthermore, removal of the race coefficient for
eGFR calculation reduced baseline eGFR in Black enrollees by approximately $10 \mathrm{~mL} / \mathrm{min} / 1.73 \mathrm{~m} \mathrm{[2].} \mathrm{Therefore,}$ use of a race coefficient likely masks real differences in CKD prevalence and hinders opportunities for timely identification and clinical management to slow CKD progression among Black enrollees.

We also found that MA enrollees with clinically recognized CKD had lower eGFR levels than patients without clinically recognized CKD, but kidney function declined more slowly in enrollees with clinical recognition. It is possible that CKD is only diagnosed once patients' CKD has progressed sufficiently to warrant closer monitoring and clinical recognition. Such clinical recognition is highly correlated with receipt of nephrology consultation and supports prior work indicating that early referral and intervention by nephrology can slow CKD progression and improve dialysis preparedness [30]. This finding also signals an opportunity to improve recognition and documentation of CKD for nonnephrology providers and improve timely referral to nephrology care. Furthermore, we found a significantly higher risk of CKD progression among younger enrollees. This may be related to more aggressive CKD diagnosed at younger ages and warrants further exploration in cohorts with a wider age distribution to facilitate more granular analysis of these trends.

Collectively, these results suggest that closer surveillance and management of individuals from ethnic and racial minority groups is warranted, and exclusion of the race coefficient with GFR estimation may be warranted. Our study further suggests that clinical recognition and documentation of CKD is a critical component to riskreduction strategies targeting CKD progression. Health system-level interventions, including electronic medical record dashboards demonstrating predicted risk of CKD progression, and integration of decision support tools to encourage appropriate management and timely specialty referral may attenuate the adverse outcomes associated with CKD progression risk.

This study is subject to several limitations. First, these results may not generalize to Medicare FFS or patients with CKD covered by other types of insurance, although the extent of selection bias has significantly mitigated in recent years as the health status of FFS and MA enrollees has become less different [31]. Second, race and ethnicity are imputed from data sourced from a national supplier of consumer marketing data using a proprietary methodology that may mischaracterize the race or ethnicity of some enrollees. Third, due to low frequency of reported albuminuria testing, our analysis included individuals with an eGFR $60-90 \mathrm{~mL} / \mathrm{min} / 1.73 \mathrm{~m}^{2}$ without albuminuria (or 
without albuminuria testing), which would be consider low risk for CKD outcomes. We were also unable to adjust for medication use associated with lower baseline eGFR and slower progression. Fourth, our study was limited to a 5-year time frame and used baseline covariates in our models; we did not evaluate time-varying covariates which may impact trajectories of CKD progression in the shorter and longer terms. Fifth, we cannot fully account for the likelihood of residual confounding, as comorbidity control and race/ethnicity are not independent of each other. Finally, a limitation of these results is the use of an imputed race/ethnicity variable based on the data from the OLDW database that likely has measurement error. Use of race/ethnicity in these analyses has implications both in the assessment of estimated GFR and as a covariate for adjustment. The race/ethnicity results should not be overinterpreted as they require validation in future work.

These results suggest that population surveillance of CKD progression and risk factor identification are important. Simple risk stratification interventions may significantly impact the poor outcomes associated with this costly and increasingly prevalent condition that increases patient risk for cardiovascular disease, ESRD, and death.

\section{Statement of Ethics}

The study was approved as exempt by the institutional review board of Duke University.

\section{Conflict of Interest Statement}

The coauthors reported receiving institutional grants from the Department of Veterans Affairs HSR\&D (M.L.M., V.W., and V.S.), the National Institute for Drug Abuse (M.L.M. and V.S.), the National Institute Diabetes and Digestive and Kidney Diseases (V.W., C.J.D., M.L.M., and V.S.), and an institutional contract from the Centers for Medicare \& Medicaid Services (M.L.M., V.W., V.S., and C.J.D.). Dr. Maciejewski reported ownership of Amgen stock due to his spouse's employment. Dr. Diamantidis reports consultancy with United Health Group. The authors report no other conflicts of interest.

\section{Funding Sources}

The research in this article was supported by the Centers for Medicare \& Medicaid Services under Contract No. HHSM-5002014-00442 G with the National Committee for Quality Assurance.

1 OptumLabs. OptumLabs and OptumLabs Data Warehouse (OLDW) Descriptions and Citation. Cambridge, MA: n.p., May 2019. PDF. Reproduced with permission from OptumLabs.
This work was also supported by the Office of Research and Development, Health Services Research and Development Service, and Department of Veterans Affairs (CIN 13-410). Dr. Maciejewski was supported by a Research Career Scientist award from the Department of Veterans Affairs (RCS 10-391). The Centers for Medicare \& Medicaid Services provided feedback on interpretation of the data when the manuscript was reviewed and approved, but had no role in the design, conduct, collection, management, or analysis of the data. The views expressed in this article are those of the authors and do not necessarily reflect the position or policy of the Department of Veteran Affairs, Duke University, the National Committee for Quality Assurance, the Centers for Medicare \& Medicaid Services, or the US Department of Health and Human Services.

\section{Author Contributions}

C.J.D., V.W., V.A.S., L.Z., and M.L.M. designed the study, wrote, and edited the manuscript, L.Z. researched and analyzed data. S.H.S. and L.T. reviewed the manuscript. All the authors approved the final version of the manuscript to be published. C.J.D. is the guarantor of this work.

\section{Data Availability Statement}

This study used deidentified administrative claims and electronic health record (EHR) data from the OptumLabs Data Warehouse (OLDW). The database contains longitudinal health information on enrollees and patients, representing a diverse mixture of ages, ethnicities, and geographical regions across the USA. The claims data in OLDW include medical and pharmacy claims, laboratory results, and enrollment records for commercial and Medicare Advantage enrollees. The EHR-derived data include a subset of EHR data that has been normalized and standardized into a single database. Study data were accessed using techniques compliant with the Health Insurance Portability and Accountability Act of 1996 (HIPAA), and because this study involved analysis of pre-existing, deidentified data, it was exempt from institutional review board approval ${ }^{1}$.

\section{References}

1 Wang V, Diamantidis CJ, Wylie J, Greer RC. Minding the gap and overlap: a literature review of fragmentation of primary care for chronic dialysis patients. BMC Nephrol. 2017;18(1):274.

2 System USRD. Annual data report: atlas of chronic kidney disease and end-stage renal disease in the United States. Minneapolis, MN: USRDS; 2016.

3 Li L, Astor BC, Lewis J, Hu B, Appel LJ, Lipkowitz MS, et al. Longitudinal progression trajectory of GFR among patients with CKD. Am J Kidney Dis. 2012;59(4):504-12.

4 Xie Y, Bowe B, Xian H, Balasubramanian S, Al-Aly Z. Estimated GFR trajectories of people entering CKD stage 4 and subsequent kidney disease outcomes and mortality. Am J Kidney Dis. 2016;68(2):219-28.

Diamantidis/Zepel/Wang/Smith/ Hudson Scholle/Tamayo/Maciejewski 
5 Caravaca-Fontan F, Azevedo L, Luna E, Caravaca F. Patterns of progression of chronic kidney disease at later stages. Clin Kidney J. 2018;11(2):246-53.

6 O'Hare AM, Batten A, Burrows NR, Pavkov ME, Taylor L, Gupta I, et al. Trajectories of kidney function decline in the 2 years before initiation of long-term dialysis. Am J Kidney Dis. 2012;59(4):513-22.

7 Neugarten J, Acharya A, Silbiger SR. Effect of gender on the progression of nondiabetic renal disease: a meta-analysis. J Am Soc Nephrol. 2000;11(2):319-29.

8 Tangri N, Grams ME, Levey AS, Coresh J, Appel LJ, Astor BC, et al. Multinational assessment of accuracy of equations for predicting risk of kidney failure: a meta-analysis. JAMA. 2016;315(2):164-74.

9 Tangri N, Inker LA, Hiebert B, Wong J, Naimark D, Kent D, et al. A dynamic predictive model for progression of CKD. Am J Kidney Dis. 2017;69(4):514-20.

10 Yang W, Xie D, Anderson AH, Joffe MM, Greene T, Teal V, et al. Association of kidney disease outcomes with risk factors for CKD: findings from the Chronic Renal Insufficiency Cohort (CRIC) study. Am J Kidney Dis. 2014;63(2):236-43.

11 Hammill BG, Curtis LH, Qualls LG, Hastings SN, Wang V, Maciejewski ML. Linkage of laboratory results to medicare fee-for-service claims. Med Care. 2015;53(11):974-9.

12 Tsai WC, Wu HY, Peng YS, Ko MJ, Wu MS Hung KY, et al. Risk factors for development and progression of chronic kidney disease: a systematic review and exploratory meta-analysis. Medicine. 2016;95(11):e3013.

13 Diamantidis CJ, Hale SL, Wang V, Smith VA, Scholle SH, Maciejewski ML. Lab-based and diagnosis-based chronic kidney disease recognition and staging concordance. BMC Nephrol. 2019;20(1):357.
14 Kawachi I, Daniels N, Robinson DE. Health disparities by race and class: why both matter. Health Aff. 2005;24(2):343-52.

15 Suarez J, Cohen JB, Potluri V, Yang W, Kaplan DE, Serper M, et al. Racial disparities in nephrology consultation and disease progression among veterans with CKD: an Observational Cohort Study. J Am Soc Nephrol. 2018; 29(10):2563-73.

16 Go AS, Chertow GM, Fan D, McCulloch CE Hsu CY. Chronic kidney disease and the risks of death, cardiovascular events, and hospitalization. N Engl J Med. 2004;351(13):1296-305.

17 Curto V, Einav L, Finkelstein A, Levin J, Bhattacharya $J$. Health care spending and utilization in public and private medicare. Am Econ J Appl Econ. 2019;11(2):302-32.

18 Park S, Larson EB, Fishman P, White L, Coe NB. Differences in health care utilization, process of diabetes care, care satisfaction, and health status in patients with diabetes in medicare advantage versus traditional medicare. Med Care. 2020;58(11):1004-12.

19 Newhouse JP, Price M, McWilliams JM, Hsu J, McGuire TG. How much favorable selection is left in medicare advantage? Am J Health Econ. 2015;1(1):1-26.

20 Newhouse JP, Price M, McWilliams JM, Hsu J, Souza J, Landon BE. Adjusted mortality rates are lower for medicare advantage than traditional medicare, but the rates converge over time. Health Aff. 2019;38(4):554-60.

21 Kadatz MJ, Lee ES, Levin A. Predicting progression in CKD: perspectives and precautions. Am J Kidney Dis. 2016;67(5):779-86.

22 OptumLabs. OptumLabs and OptumLabs Data Warehouse (OLDW) Descriptions and Citation. Cambridge, MA: OptumLabs; 2019.
23 Cromartie J . Rural-urban commuting area codes. U.S. department of agriculture. Data products web site. 2020. https://www.ers. usda.gov/data-products/rural-urban-commuting-area-codes/ Accessed 2020 Jul 20.

24 Levey AS, Stevens LA. Estimating GFR using the CKD epidemiology collaboration (CKDEPI) creatinine equation: more accurate GFR estimates, lower CKD prevalence estimates, and better risk predictions. Am J Kidney Dis. 2010;55(4):622-7.

25 Leffondre K, Boucquemont J, Tripepi G, Stel VS, Heinze G, Dunkler D. Analysis of risk factors associated with renal function trajectory over time: a comparison of different statistical approaches. Nephrol Dial Transplant. 2015; 30(8):1237-43.

26 Delgado C, Baweja M, Burrows NR, Crews DC, Eneanya ND, Gadegbeku CA, et al. Reassessing the inclusion of race in diagnosing kidney diseases: an interim report from the NKF-ASN task force. Am J Kidney Dis. 2021; 78(1):103-15.

27 Young BA, Katz R, Boulware LE, Kestenbaum B, de Boer IH, Wang W, et al. Risk factors for rapid kidney function decline among African Americans: the Jackson Heart Study (JHS). Am J Kidney Dis. 2016;68(2):229-39.

28 Tangri N, Stevens LA, Griffith J, Tighiouart $\mathrm{H}$, Djurdjev O, Naimark D, et al. A predictive model for progression of chronic kidney disease to kidney failure. JAMA. 2011;305(15): 1553-9.

29 Chen TK, Knicely DH, Grams ME. Chronic kidney disease diagnosis and management: a review. JAMA. 2019;322(13):1294-304.

30 Smart NA, Dieberg G, Ladhani M, Titus T. Early referral to specialist nephrology services for preventing the progression to end-stage kidney disease. Cochrane Database Syst Rev. 2014;6:CD007333.

31 Newhouse JP, Price M, McWilliams JM, Hsu J, McGuire TG. How much favorable selection is left in medicare advantage? Am J Health Econ. 2015;1(1):1-26. 